\title{
ANÁLISIS DEL MOVIMIENTO EN RELACIÓN CON LA ESTRUCTURA MUSICAL DEL FONDU Y DEL DOUBLE FONDU A $45^{\circ}$
}

\author{
África Hernández Castillo \\ Investigadora independiente, Prof. Danza Clásica
}

\section{Resumen}

En este artículo se establece el análisis del movimiento de los pasos de la danza académica fondu y double fondu a $45^{\circ}$ en compás binario y ternario. Se exponen las diversas dinámicas posibles para concluir con el adecuado acompañamiento musical durante su ejecución y la coordinación final para su realización a $45^{\circ}$.

Palabras clave: FONDU; DOUBLE FONDU; COMPÁS BINARIO; COMPÁS TERNARIO; METODOLOGÍA DANZA ACADÉMICA

\section{ANALYSIS OF THE MOVEMENT IN RELATION TO THE STRUCTURE MUSICAL OF THE FONDU AND THE DOUBLE FONDU AT $45 \stackrel{0}{ }$}

\section{Abstract}

In this essay, the movement of the academic dance steps fondu and double fondu at $45^{\circ}$ will be explained in binary and ternary meters. Different dinamics will be exposed, concluding with the most suitable musical accompaniment for its execution, as well as the most suitable coordination for its performance at $45^{\circ}$.

Keywords: FONDU; DOUBLE FONDU; BINARY METER; TERNARY METER; METHODOLOGY OF ACADEMIC DANCE

\footnotetext{
Hernández Castillo, África. 2019. "Análisis del movimiento en relación con la estructura musical del fondu y del double fondu a 45 ”". AusArt 7 (1): 171-181. DOI: 10.1387/ausart.20656
}

\section{AUSART}




\section{INTRODUCCIÓN}

Desde que me adentré en el campo de la investigación de la metodología de la danza académica, pude observar que el mayor número de profesores de danza clásica, al marcar los pasos básicos, lo hacemos de una manera similar aparentemente. Pero si analizamos minuciosamente el movimiento de los pasos, es cuando se puede apreciar una gran diversidad en su realización.

La comunicación que presenté en el V Congreso Nacional y II Internacional Danza más Investigación celebrado en Sevilla en noviembre de 2018, "Análisis en compás binario y ternario de contenidos derivados del rond de jambe par terre", es lo que me motivó a adentrarme en este artículo, por la controversia que plantea también el análisis del fondu y el double fondu en compás binario y ternario.

Una vez más se encontró una escasez de documentación escrita, y la consultada no alcanzaba la exactitud analítica requerida para poder hacer un estudio comparativo profundo. Este fue otro de los motivos que me llevó a investigar la ejecución precisa de estos pasos.

\section{OBJETIVO}

Uno de mis objetivos fue analizar el movimiento de ambos pasos en compás binario y ternario, y partiendo de ese análisis, investigar y contrastar los resultados con su ejecución por los profesores consultados y con las percepciones extraídas durante el visionado de clases de danza.

\section{Proceso de ENSEÑAnZA-APREndizaje del FONDU Y DEL DOUBLE FONDU}

En mis publicaciones realicé el siguiente proceso de enseñanza-aprendizaje, el cual está basado en el de la Hungarian Dance Academy de Budapest, según el método Vaganova:

- 1er Curso

- Fondu a $45^{\circ}$, inicialmente de frente a la barra y luego de perfil a la barra en 2 compases de 4/4. 
- $2^{\circ}$ Curso

- Fondu a $45^{\circ}$ en demi-pointe en face, y a pie plano en Pequeñas Poses, en 2 compases de 4/4.

- Double fondu a $45^{\circ}$, se introduce en 4 compases de $4 / 4$ de perfil a la barra, para continuar su práctica en 2 compases de 4/4 en demi-pointe en face, y a pie plano en Pequeñas Poses.

- 3er Curso

- Fondu a $45^{\circ}$ en demi-pointe en Pequeñas Poses en 2 compases de $4 / 4$ y luego en 1 compás de $4 / 4$.

- Double fondu a $45^{\circ}$ en demi-pointe en Pequeñas Poses en 2 compases de 4/4 y luego en 1 compás de 4/4.

A pesar de que estos pasos continuarán desarrollándose a lo largo de los siguientes cursos (en su ejecución a $90^{\circ}$ a pie plano y en demi-pointe en face y en Grandes Poses, con tombé a $45^{\circ}$ y a $90^{\circ}$ en face y en Pequeñas y Grandes Poses, en tournant a $45^{\circ}$ y a $90^{\circ}$ en face y en Pequeñas y Grandes Poses, etc.), he centrado mi investigación en su realización en 1 compás de 4/4, y desarrollaré su análisis en 2 compases de 3/4.

\section{ANÁLisis deL MOVIMIENTO REALIZAdO EN MIS PUBLICACIONES DEL FONDU Y DOUBLE FONDU EN 1 COMPÁS DE $4 / 4$ (NEGRA $=74$, ANDANTE)}

- Fondu en 1 compás de 4/4

- 1er Compás:

- 1-2: Sur le cou de pied en demi-plié, brazo a $1^{a}$ posición.

- 3-4: Estiraremos la pierna de base a la vez que la de acción se extenderá a $45^{\circ}$, brazo a $2^{a}$ posición. En el caso de hacer otro fondu, en el tiempo 4 haremos allongé.

- Double fondu en 1 compás de 4/4

- 1er Compás:

- 1: Sur le cou de pied en demi-plié. brazo a $1^{a}$ posición.

- 2: Estiraremos la pierna de base manteniendo la de acción sur le cou de pied.

- 3: La pierna de base hace demi-plié.

- 4: Estiraremos la pierna de base a la vez que la de acción se extenderá a $45^{\circ}$, brazo a $2^{a}$ posición. En el caso de hacer otro double fondu, en el segundo 1/8 del tiempo 4 haremos allongé. 


\section{ANÁlisis del MOVIMiENTO dEL FONDU y dOUBLE FONDU EN 2 COMPASES \\ DE $3 / 4 \quad($ NEGRA $=110$, ALLEgRO $)$}

Debido al debate que implica la conveniencia de su ejecución en compás binario o ternario, se decide hacer el estudio en 2 compases de 3/4 bajo un criterio personal, por no haber encontrado ninguna publicación con su análisis en compás ternario.

- Fondu en 2 compases de 3/4

- 1er Compás:

- 1-2-3: Sur le cou de pied en demi-plié, brazo a $1^{a}$ posición.

- $2^{\circ}$ Compás:

- 1-2-3: Estiraremos la pierna de base a la vez que la de acción se extenderá a $45^{\circ}$, brazo a $2^{\mathrm{a}}$ posición. En el caso de hacer otro fondu, en el tiempo 3 haremos allongé.

- Double fondu en 2 compases de 3/4

Se establecen dos maneras de llevarse a cabo:

A)Esta forma, es la que he hecho a lo largo de mi carrera profesional como bailarina. Por lo que se podría decir que ha sido la realizada por reproducción de una enseñanza.

Se comienza el movimiento sur le cou de pied en demi-plié con el brazo a $1^{\text {a }}$ posición, en el tiempo 3 del compás anterior (en la anacrusa).

- 1er Compás:

»1: Estiraremos la pierna de base manteniendo la de acción sur le cou de pied.

2: Pausa.

»3: La pierna de base hace demi-plié.

- $2^{\circ}$ Compás:

»1: estiraremos la pierna de base a la vez que la de acción se extenderá a $45^{\circ}$, brazo a $2^{\mathrm{a}}$ posición.

"2: Pausa, o en el caso de hacer otro double fondu, haremos allongé.

» 3: Se realizará el primer movimiento del siguiente double fondu. 
B) Esta forma está basada en la teoría de que el fondu hace referencia al descenso sobre una pierna en demi-plié de manera legatto, pudiéndose relacionar directamente con las estructuras del análisis del movimiento del salto tanto en su fase inicial como en la final.

- 1er Compás:

»1-2: Sur le cou de pied en plié. brazo en $1^{\text {a }}$ posición.

»3: Estiraremos la pierna de base manteniendo la de acción sur le cou de pied.

- $2^{\circ}$ Compás:

》1-2: La pierna de base hace demi-plié.

"3: Estiraremos la pierna de base a la vez que la de acción se extenderá a $45^{\circ}$, brazo a $2^{\mathrm{a}}$ posición. En el caso de hacer otro double fondu, en el segundo $1 / 8$ del tiempo 3 haremos allongé.

Se observa que en el fondu en 2 compases de 3/4, sigue existiendo un movimiento legatto y regular. Pero en el double fondu, en la forma A) se percibe que hay un impulso en el tiempo fuerte del compás. Y la forma $B$ ) está más relacionada con el movimiento continuo, gradual, ligado y suave del fondu; ya que si acentuamos el movimiento arriba, se podría confundir con la dinámica del ballonné en demi-pointe o sur la pointe.

\section{METODOLOGÍA}

Una vez planteado el análisis del movimiento en relación con la estructura musical del fondu y double fondu en compás binario y ternario desde mi punto de vista personal, me adentré en la investigación observando una gran diversidad de ejecución de estos pasos por otros maestros y discentes a través de entrevistas personales, observación de clases y material audiovisual. 


\section{RESULTADOS}

- Fondu en 1 compás de $4 / 4$

- Hay una coincidencia entre el material bibliográfico y audiovisual consultado basado en el sistema Vaganova, y mi propuesta.

- Respecto al análisis de la ejecución realizada por los profesores y alumnos de danza, se observan diferentes maneras de hacerlo:

a) 1-2: Sur le cou de pied en demi-plié.

3-4: Estiraremos la pierna de base a la vez que la de acción se extenderá a $45^{\circ}$, brazo a $2^{\mathrm{a}}$ posición.

b) 1: Sur le cou de pied en demi-plié.

2: Pausa.

3: Attitude en demi-plié.

4: Estiraremos la pierna de base a la vez que la de acción se extenderá a $45^{\circ}$, brazo a $2^{\mathrm{a}}$ posición.

c) 1: Sur le cou de pied en demi-plié.

2: Pausa.

3-4: Estiraremos la pierna de base a la vez que la de acción se extenderá a $45^{\circ}$, brazo a $2^{\mathrm{a}}$ posición.

d) 1: Sur le cou de pied en demi-plié.

2: Pausa.

3: Estiraremos la pierna de base a la vez que la de acción se ex- tenderá a $45^{\circ}$, brazo a $2^{\mathrm{a}}$ posición.

4: Pausa, o el allongé pertinente.

e) 1: Sur le cou de pied en demi-plié.

2: Attitude en demi-plié.

3: Estiraremos la pierna de base a la vez que la de acción se extenderá a $45^{\circ}$, brazo a $2^{\mathrm{a}}$ posición.

4: Pausa.

f) 1: Sur le cou de pied en demi-plié.

2: Attitude en demi-plié.

3: Estiraremos la pierna de base a la vez que la pierna de acción se ex-

tenderá a $45^{\circ}$, brazo a $2^{\mathrm{a}}$ posición.

4: Comienzan a doblarse ambas piernas. 
- Double fondu en 1 compás de 4/4

- Una vez más, en las consultas realizadas que se basan en la escuela rusa, el modo de realización coincide con mi planteamiento.

- También se advierte en los docentes y discentes de danza diferentes maneras de ejecución:

a) 1: Sur le cou de pied en demi-plié.

2: Estiraremos la pierna de base manteniendo la de acción sur le cou de pied.

3: La pierna de base hace demi-plié.

4: Estiraremos la pierna de base a la vez que la de acción se extenderá a $45^{\circ}$, brazo a $2^{\mathrm{a}}$ posición.

b) En la anacrusa se inicia el sur le cou de pied en demi-plié. 1: Estiraremos la pierna de base manteniendo la de acción sur le cou de pied.

2: La pierna de base hace demi-plié.

3: Estiraremos la pierna de base a la vez que la de acción se extenderá a $45^{\circ}$, brazo a $2^{\mathrm{a}}$ posición.

4: De nuevo se inicia el double fondu con sur le cou de pied en demi-plié

En el double fondu en 1 compás de 4/4, se han encontrado básicamente dos estructuras, aunque con una gran diversidad en el comportamiento de los brazos y la cabeza. Y pude apreciar que se realizaba de la misma manera durante su práctica en cruz, utilizando una negra para cada uno de los 4 movimientos, aunque unos comenzaban en la anacrusa y otros en el tiempo fuerte del compás.

- Fondu en 2 compases de 3/4

- No he podido encontrar el análisis en compás ternario en ninguna publicación consultada.

- Durante la investigación en la webgrafía de la Escuela Vaganova, no he visto el uso del compás ternario en este paso.

- Respecto a los profesores y alumnos de danza, pude observar diversas maneras de realizarlo:

a) 1-2-3: Sur le cou de pied en demi-plié.

1-2-3: Estiraremos la pierna de base a la vez que la de acción se extenderá a $45^{\circ}$, brazo a $2^{\mathrm{a}}$ posición. 
b) 1-2: Sur le cou de pied en demi-plié.

3: Attitude en plié.

1-2: Estiraremos la pierna de base a la vez que la de acción se extenderá a $45^{\circ}$, brazo a $2^{\mathrm{a}}$ posición.

3: Allongé.

c) 1: Sur le cou de pied en demi-plié.

2: Pausa.

3: Attitude en plié.

1: Estiraremos la pierna de base a la vez que la de acción se extenderá a $45^{\circ}$, brazo a $2^{\mathrm{a}}$ posición.

2-3: De nuevo se inicia el fondu con sur le cou de pied en demi-plié.

d) 1: Sur le cou de pied en demi-plié.

2-3: Attitude en plié.

1-2: Estiraremos la pierna de base a la vez que la pierna de acción se extenderá a $45^{\circ}$, brazo a $2^{\mathrm{a}}$ posición.

3: Comenzarán a doblarse las dos piernas.

e) 1-2: Sur le cou de pied en demi-plié.

3: Attitude en plié.

1-2: Estiraremos la pierna de base a la vez que la de acción se extenderá a $45^{\circ}$, brazo a $2^{a}$ posición.

3: Comenzarán a doblarse las dos piernas.

f) 1-2: Sur le cou de pied en demi-plié.

3: Attitude en plié.

1: Estiraremos la pierna de base a la vez que la de acción se extenderá a $45^{\circ}$, brazo a $2^{\mathrm{a}}$ posición.

2: Pausa.

3: Comenzarán a doblarse las dos piernas.

- Double fondu en 2 compases de 3/4

- No se ha encontrado el análisis en ninguna bibliografía.

- En el visionado de las clases de la Escuela Vaganova, tampoco se encontró su realización.

- Percibí una gran variedad en la realización de profesores y alumnos, pero todos iniciaban el primer movimiento del double fondu, es decir, sur le cou de pied en demi-plié en el tiempo 3 del compás anterior (anacrusa). 
a) 1: Estiraremos la pierna de base manteniendo la de acción sur le cou de pied.

2: Pausa.

3: La pierna de base hace demi-plié.

1-2: Estiraremos la pierna de base a la vez que la de acción se extenderá a $45^{\circ}$, brazo a $2^{a}$ posición.

3: Se realizará el primer movimiento del siguiente double fondu.

b) 1-2-3: Como el 1er Compás de a).

1: Estiraremos la pierna de base a la vez que la de acción se extenderá a $45^{\circ}$, brazo a $2^{\mathrm{a}}$ posición.

2: Pausa.

3: Se realizará el primer movimiento del siguiente double fondu.

c) 1-2: Estiraremos la pierna de base manteniendo la de acción sur le cou de pied.

3: La pierna de base hace demi-plié.

1-2-3: Como el $2^{\circ}$ Compás de a).

d) 1: Estiraremos la pierna de base manteniendo la de acción sur le cou de pied.

2: La pierna de base hace demi-plié.

3: Attitude en plié.

1: Estiraremos la pierna de base a la vez que la de acción se extenderá a $45^{\circ}$, brazo a $2^{a}$ posición.

2: Pausa.

3: Se realizará el primer movimiento del siguiente double fondu.

e) 1-2-3: Como el $2^{\circ}$ Compás de a), deberá ser Como el 1er Compás de c).

1: Estiraremos la pierna de base a la vez que la de acción se extenderá a $45^{\circ}$, brazo a $2^{a}$ posición.

2: Comenzarán a doblarse ambas piernas.

3: Se realizará el primer movimiento del siguiente double fondu.

f) 1-2-3: Como el $2^{\circ}$ Compás de a).

1: Estiraremos la pierna de base a la vez que la de acción se extenderá a $45^{\circ}$, brazo a $2^{a}$ posición.

2: Pausa. 
3: Se realizará el primer movimiento del siguiente double fondu.

En la Forma c), es donde se puede apreciar que el movimiento legatto se produce al estirar las piernas y no al hacer demi-plié, atendiendo los tiempos fuertes y débiles característicos del 3/4.

\section{CONCLUSIONES}

Nos encontramos con dos pasos, cuya realización está compuesta por dos movimientos (en el caso del fondu), y de cuatro movimientos (en el del double fondu), por lo que se podría concretar que el compás binario sería el idóneo para su acompañamiento musical.

La realización de cada contenido en 1 compás de 4/4, otorga la fluidez y el movimiento continuo y ligado que caracteriza a los dos pasos, de manera uniforme y sin pausas, junto a una perfecta sincronización de ambas piernas.

Durante el proceso de investigación se percibe tanto en la mayoría de los encuestados como en las clases visionadas, la preferencia por la realización en compás ternario y que su ejecución en compás binario va sujeta al acompañamiento musical de un tango o habanera. La mayoría atienden en su realización a los tiempos fuertes del compás en lugar de al carácter propio del fondu y double fondu, y que la forma de ejecución de ambos contenidos, arrojaba una diversidad de resultados. Si a todas estas formas de ejecución se le suma las distintas posibilidades en la coordinación del port de bras, se obtendría un mayor número de variantes en su ejecución.

La mayor parte de los colaboradores, no mantenían la misma estructura de realización durante su práctica en cruz. En otros, el movimiento de las piernas era staccato y donde se apreciaba el movimiento legatto era en el port de bras.

Se ha observado una clara igualdad del análisis del movimiento en relación con la estructura musical en compás binario del fondu y del double fondu entre la escuela Vaganova y mi propuesta personal. 
Se concluye que el profesor, dependiendo de su objetivo, deberá elegir con conciencia la manera de ejecución de estos pasos: su realización con una acentuación hacia arriba (como entrenamiento de los pasos que requieran la dinámica del plié-rélevé); o con un movimiento continuo y fluido hacia abajo, es decir, enfatizando el demi-plié (para la ejecución correcta y elástica de los saltos). Se deberá también tener en cuenta la velocidad metronómica y los otros pasos con los que se vaya a combinar.

\section{Referencias bibliográficas}

Hernández Castillo, África. 2009. La danza académica y su metodología: Análisis del movimiento en relación con la estructura musical: Nivel elemental. Valencia: Mahali

Kostrovitskaya, Vera \& Alexei Pisarev. 1995. School of classical dance. Traductor John Barker. London: Dance Books

Tarassow, Lew Wassiljewitsch. 1994. Klassischer Tanz: Die Schule des Tänzers. Trad. M. Puttke. Berlín: Henschel

Vaganova Ballet Academy. 2008. "3rd grade, Novitskaya/Gortchakova [teachers], part 1". Vídeo de Youtube, 7:05. https://www.Youtube.com/watch?v=WaKKCEWC-rQ

Vaganova Ballet Academy. 2015. "Barre, Classical dance exam: Girls, 4th class". Teacher, Ludmila Komolova. Vídeo de Youtube, 11:36. https://www.Youtube.com/watch?v=hi359cfO0-I

Vaganova Ballet Academy. 2016. "Classical dance exam: Girls, 5th class". Teacher, María Gribanova. Vídeo de Youtube, 52:19. https://www.Youtube.com/watch?v=8RtsRyDyeZk

Vaganova, Agrippinalakovlevna. 1945. Las bases de la danza clásica: Ciento veinte dibujos explicativos. Trad. M. Ourvantzoff. Buenos Aires: Centurión

Zsuzsa, Merényi.1990. A klasszikus balett módszertana. II. rész. Budapest: Tankönyvkiadó [Metodología del Ballet Clásico, $1^{a}, 2^{a}$ y $3^{a}$ sección del $1 \mathrm{er}$ al $8^{\circ}$ grado] 